\title{
Acute Aortic Dissection Presenting with Primarily Abdominal Pain: A Rare Manifestation of a Deadly Disease
}

\author{
Gilbert R. Upchurch, Jr., $M D,{ }^{1}$ Christoph Nienaber, $M D^{2}{ }^{2}$ Rossella Fattori, $M D,{ }^{3}$ \\ Arturo Evangelista, $M D{ }^{4}$ Jae Oh, $M D{ }^{5}$ Jeanna V. Cooper, $M S^{1}{ }^{1}$ Eric Isselbacher, $M D,{ }^{6}$ \\ Toru Suzuki, MD, ${ }^{7}$ and Kim A. Eagle, MD, ${ }^{1}$ for the IRAD Investigators, Ann Arbor, Michigan, \\ Rochester, Minnesota, Boston, Massachusetts, USA, and Rostock, Germany, Bologna, Italy, \\ Barcelona, Spain, and Tokyo, Japan
}

\begin{abstract}
The objective of this study was to determine the morbidity and mortality of patients with acute thoracic aortic dissections who present primarily with abdominal pain. Nine hundred ninety-two patients (mean age, 62.1 years $\pm 14.1 ; 68 \%$ male) encountered from 1996 to 2001 with acute thoracic aortic dissections from the International Registry of acute Aortic Dissection were studied. Patient demographics, presenting symptoms, signs of aortic dissection, aortic pathology, and mortality were compared in patients presenting primarily with abdominal pain (group I, 46 patients, $4.6 \%$ ) versus all others (group II). Demographics were similar between the two groups. When signs of aortic dissection were examined, $63 \%$ of patients in group I presented with hypertension compared to only $47 \%$ of patients in group II $(p=0.04)$. Patients in group I were less likely to present with evidence of end-organ malperfusion. Importantly, mortality in patients with a type B dissection, specifically following surgery for the dissection, was significantly increased in patients who presented primarily with abdominal pain (group I, $28 \%$ mortality vs. group II, $10.2 \%$ mortality; $p=0.02$ ). This study documented increased mortality in patients with acute thoracic aortic dissections who present primarily with abdominal pain, underscoring the importance of maintaining a high index of suspicion for an aortic dissection in patients who have appropriate risk factors.
\end{abstract}

\section{INTRODUCTION}

Diseases of the aorta, including aortic dissection, were the 14th leading cause of death in the United States in 1999. ${ }^{1}$ Despite this alarming figure, acute thoracic aortic dissection continues to be one of the most commonly missed diagnoses resulting in high mortality rates. This is believed secondary to its

\footnotetext{
${ }^{1}$ University of Michigan Health System, Ann Arbor, MI, USA.

${ }^{2}$ University of Rostock, Rostock, Germany.

${ }^{3}$ University Hospital S. Orsola, Bologna, Italy.

${ }^{4}$ Hospital General Universitari Vall d'Hebron, Barcelona, Spain.

${ }^{5}$ Mayo Clinic, Rochester, MN, USA.

${ }^{6}$ Massachusetts General Hospital, Boston, MA, USA.

${ }^{7}$ University of Tokyo, Tokyo, Japan.

Correspondence to: Gilbert R. Upchurch, Jr., MD, University of Michigan Hospital-2210 THCC, 1500 East Medical Center Drive, Ann Arbor, MI 48109-0329,USA, E-mail: riversu@umich.edu

Ann Vasc Surg 2005; 19: 367-373

DOI: $10.1007 / s 10016-004-0171-x$

(C) Annals of Vascular Surgery Inc.

Published online: April 4, 2005
}

variable presentation, its lack of a reliable serum biomarker, and the delay in time to diagnosis. A recent comprehensive review including 274 potential sources suggested that the presence of pulse deficits or a focal neurologic deficit increased the likelihood of diagnosing an acute thoracic dissection. ${ }^{2}$ Conversely, the presence of a normal chest $\mathrm{x}$ ray or the absence of pain lowered the likelihood that a patient had an acute aortic dissection.

While the typical patient with an acute thoracic aortic dissection presents with complaints of a sharp, tearing pain in the chest or back, ${ }^{3}$ this constellation of symptoms is not always present. As such, many patients with acute aortic dissections are relegated to step down units and discharged home following normal serial EKGs and troponins for a suspected coronary source of their pain.

Nonlocalizing symptoms with pain in various locations and to various degrees are not uncommon in this cohort of patients, resulting in the diagnosis of a thoracic aortic dissection only being suspected in as few as $15 \%$ to $43 \%$ who present 
Table I. Patient demographics

\begin{tabular}{llrr}
\hline Factor & Group I $(N=46)[n(\%)]$ & Group II $(N=946)[n(\%)]$ & $p$ \\
\hline Age $($ mean \pm SD) & $63.4 \pm 12.8$ & $62 \pm 14.2$ & 0.53 \\
Hypertension & $31(73.8)$ & $659(71.1)$ & 0.70 \\
Marfan's syndrome & $2(4.7)$ & $48(5.2)$ & $>0.99$ \\
Atherosclerosis & $13(30.2)$ & $257(27.9)$ & 0.74 \\
Diabetes & $1(2.4)$ & $39(4.3)$ & 0.57 \\
Known aortic aneurysm & $2(4.7)$ & $130(14.1)$ & 0.08 \\
Prior aortic dissection & $5(11.6)$ & $49(5.3)$ & 0.09 \\
Bicuspid aortic valve $(N=531)$ & $0(0)$ & $18(3.5)$ & $>0.99$ \\
Aortic valve disease & $2(4.8)$ & $77(8.6)$ & 0.57 \\
\hline
\end{tabular}

acutely $^{4,5}$ and a delay in diagnosis of $>24$ hr in close to $40 \%$ of patients. ${ }^{6}$ To make matters worse, it appears that many patients who have signs and symptoms consistent with an acute thoracic aortic dissection do not always have the disease. ${ }^{7}$

One of the many atypical presentations of a patient with an acute thoracic aortic dissection is the individual who presents with primary or isolated abdominal pain. While described in isolated case reports, ${ }^{8}$ to date no large series has examined this group of patients. Therefore, the objective of the current study was to use a large, international experience to determine whether patients with acute thoracic aortic dissections presenting with abdominal pain as their primary symptom had a higher morbidity and mortality compared to others presenting with a dissection.

\section{PATIENTS AND METHODS}

Nine hundred ninety-two patients (mean age, 62.1 years $\pm 14.1 ; 68 \%$ male) encountered from 1996 to 2001 with acute thoracic aortic dissections from the International Registry of acute Aortic Dissection (IRAD) were studied. ${ }^{3}$ IRAD consists of 15 international referral centers in which hospital records of patients with acute aortic dissections are assessed and reviewed by physicians. Patient demographics, presenting symptoms, signs of aortic dissection, aortic pathology, and mortality were compared in patients presenting primarily with abdominal pain (group I) versus all others (group II). Group I consisted of patients with abdominal pain only $(N=23)$ and a group of patients who had abdominal pain as their primary symptom but also stated they had chest pain $(N=23)$. The medical and surgical management of aortic dissections was determined at each individual center participating in IRAD. Descriptive statistics are described as mean \pm standard deviation. Categorical data were compared using chi-squared analysis or Fisher's exact test when appropriate. Continuous data were compared using Student's $t$-test. Life-table analysis was performed with a log-rank test. Statistical significance was assigned with $p$ values $<0.05$.

\section{RESULTS}

Traditional risk factors associated with aortic dissection, including a history of hypertension, were common in both groups (Table I). Marfan's syndrome, known to predispose patients to aortic dissection, occurred in $4.7 \%$ and $5.2 \%$ of patients in group I and group II, respectively. The presence of a known aortic aneurysm or a history of prior aortic dissection was also not statistically different between the two groups. While there were no statistical differences in the nature of the pain that patients presented with, it is notable that the mean time to diagnosis of an acute aortic dissection was $84.4 \mathrm{hr}$ in group I compared to $50.4 \mathrm{hr}$ in group II (Table II).

Despite the fact that there were no differences in the two groups in the percentage of patients who had a history of hypertension, patients in group I more often presented acutely with hypertension ( $p=$ 0.04) (Table III). In addition, there was a decreased incidence of end-organ malperfusion, including pulse deficits, cerebrovascular accidents, and ischemic lower extremities, in patients with primarily abdominal pain (group I). When type (A vs. B) and management (medical versus surgical) of the aortic dissections were examined, keeping with the standard of care, the majority of patients with type A dissections were managed surgically, whereas patients with type $\mathrm{B}$ dissections were most often managed medically (Table IV).

Overall in-hospital mortality was not different between the two groups $(26.1 \%$ for group I vs. $22.9 \%$ for group II, $p=0.62$; Table V). However, in-hospital mortality in patients with type B aortic dissections was significantly higher in group I 
Table II. Presenting symptoms

\begin{tabular}{lccc}
\hline Symptom & Group I $[n(\%)]$ & Group II $[n(\%)]$ & $p$ \\
\hline Migrating pain & $9(20)$ & $168(18.2)$ & 0.76 \\
Radiating pain & $12(27.9)$ & $332(35.7)$ & \\
Quality of pain & & & \\
Tearing & $9(25)$ & $287(38.6)$ & 0.3 \\
Sharp & $16(44.4)$ & $335(45.1)$ & 0.1 \\
Pressure & $12(33.3)$ & $202(27.2)$ & 0.42 \\
Burning & $2(5.6)$ & $83(9.8)$ & 0.57 \\
Abrupt onset & $39(84.8)$ & $511(74.5)$ & 0.41 \\
Presenting within 6 hr of symptom onset & $28(82.4)$ & $17.2 \pm 44.2$ & 0.3 \\
Hours from symptom onset to presentation (mean \pm SD) & $20.2 \pm 48.4$ & $50.4 \pm 97.8$ & 0.7 \\
Hours from symptom onset to diagnosis (mean \pm SD) & $84.4 \pm 193.2$ & 0.32 \\
\hline
\end{tabular}

Table III. Signs of aortic dissection

\begin{tabular}{lcrr}
\hline Presenting hemodynamics & Group I $[n(\%)]$ & Group II $[n(\%)]$ & $p$ \\
\hline Hypertensive & $27(62.8)$ & $422(46.5)$ & 0.04 \\
Normotensive & $11(24.4)$ & $353(38.8)$ & 0.05 \\
Hypotensive & $7(16.3)$ & $94(10.3)$ & 0.21 \\
Shock & $5(11.6)$ & $84(9.2)$ & 0.59 \\
Cardiac tamponade & $1(2.2)$ & $34(3.7)$ & $>0.99$ \\
First BP systolic (mean \pm SD) & $150.8 \pm 49.6$ & $82.5 \pm 42.4$ & 0.27 \\
First BP diastolic (mean \pm SD) & $85.7 \pm 25.6$ & $301(34.7)$ & 0.38 \\
Murmur of aortic insufficiency & $12(27.3)$ & $236(28.4)$ & 0.31 \\
Pulse deficits & $5(13.2)$ & $43(4.7)$ & 0.04 \\
CVA & $0(0)$ & $90(9.9)$ & 0.25 \\
Coma/altered consciousness & $2(4.8)$ & $37(4.1)$ & 0.42 \\
CHF & $2(5)$ & $79(8.7)$ & 0.68 \\
Ischemic lower extremity & $0(0)$ & 0.05 \\
\hline
\end{tabular}

$\mathrm{BP}$, blood pressure; CHF, congestive heart failure; CVA, cerebrovascular accident.

$(28 \%)$ than in group II $(10.2 \%, p=0.02)$. The increased mortality in this subset of patients was attributable to the extremely high mortality $(100 \%)$ in group I patients with type B dissections who required surgical repair of their dissection (Table V). Specifically in group I, the causes of death in the type B group included two patients who died of aortic rupture and one of visceral ischemia in the medically managed group. In the surgically managed group, two patients died following aortic rupture and two of nonspecified causes. In addition, when patients with abdominal pain as their only presenting symptom $(N=23)$ were examined separately, their mortality was still significantly higher $(28 \%, p=0.04)$ than that of group II $(11 \%)$ if they had sustained a type B dissection. In-hospital complications, specifically new neurologic deficits, cardiac tamponade, and limb ischemia, were markedly lower in group I than in group II (all $p<0.05$ ), perhaps secondary to the increased numbers of patients undergoing surgical repair in group II (Table VI).
Short-term follow-up as demonstrated by Kaplan-Meier survival curves documented a statistically significant (log-rank test, $p=0.003$ ) increased in-hospital mortality rate in patients with type B dissections who presented primarily with abdominal pain (Fig. 1). In contrast, patients with type A dissections had on average a lower mortality if they presented primarily with abdominal pain (Fig. 2).

\section{DISCUSSION}

This study documents increased mortality in patients presenting primarily with abdominal pain, especially in patients who require surgery in the setting of an acute type B thoracic aortic dissection. Unfortunately, this increased mortality occurs in the setting of few differences in patient demographics, quality or abruptness of symptoms, or signs of dissection. This paucity of signs and symptoms heralding an acute thoracic aortic dis- 
Table IV. Aortic pathology

\begin{tabular}{llrl}
\hline & Group I $[n(\%)]$ & Group II [n (\%)] & $p$ \\
\hline Total & $46(4.6)$ & $946(95.4)$ & 0.02 \\
Type A & $21(45.7)$ & $593(62.7)$ & 0.004 \\
Type B & $25(54.3)$ & $353(37.3)$ & \\
Medical (all) & $29(63)$ & $394(41.6)$ & 0.04 \\
Surgical (all) & $17(37)$ & $105(17.7)$ & $>0.99$ \\
Medical (type A) & $8(38.1)$ & $488(82.3)$ & \\
Surgical (type A) & $13(61.9)$ & $289(81.9)$ & \\
Medical (type B) & $21(84)$ & $64(18.1)$ & \\
Surgical (type B) & $4(16)$ & & \\
\hline
\end{tabular}

Table V. In-hospital mortality

\begin{tabular}{lcrr}
\hline & Group I $[n(\%)]$ & Group II [n (\%)] & $p$ \\
\hline Mortality (overall) & $12(26.1)$ & $217(22.9)$ & 0.62 \\
Mortality (type A) & $5(23.8)$ & $181(30.5)$ & 0.51 \\
Mortality (type B) & $7(28)$ & $36(10.2)$ & 0.02 \\
Mortality (surgical) & $4(23.5)$ & $138(25)$ & $>0.99$ \\
Surgical (type A) & $0(0)$ & $123(25.2)$ & 0.05 \\
Surgical (type B) & $4(100)$ & $75(23.4)$ & 0.005 \\
Mortality (medical) & $8(27.6)$ & $58(55.2)$ & 0.33 \\
Medical (type A) & $5(62.5)$ & $21(7.3)$ & $>0.99$ \\
Medical (type B) & $3(14.3)$ & $10.7 \pm 24.5$ & 0.22 \\
Days from symptom onset & $39.5 \pm 108.7$ & 0.4 \\
to death (mean \pm SD) & & & \\
\hline
\end{tabular}

section is associated with a delay in diagnosis in this subset of patients.

One of the difficulties in diagnosing patients with aortic dissections is the significant variation in presenting physical signs and symptoms, which often confuse physicians when evaluating this complex group of patients. ${ }^{5,6}$ This is compounded by the observation that there are no serum biomarkers available to accurately rule out an acute aortic dissection. This lack of a specific biomarker for acute aortic dissection may add to the complexity of making a diagnosis in these patients, as type A dissections may experience coronary artery ostial compromise with subsequent myocardial ischemia and a troponin leak. In the present study, even nonspecific serum markers of end-organ ischemia, such as acidosis, were not helpful.

Possible vascular etiologies easily ruled out in patients presenting with primarily abdominal pain include a ruptured or symptomatic abdominal aortic aneurysm (AAA). Because patients with AAAs share many of the same risk factors as those of patients with aortic dissections, namely male gender, increased age, hypertension, and Marfan's syndrome, these patients will traditionally undergo emergent abdominal and pelvic computed tomog- raphy (CT) scans to rule out an AAA, without obtaining a chest CT. This practice obviously misses type A or type B dissections, which remain confined to the chest.

Studies have suggested that thoracic aortic dissections and AAAs are rarely found concurrently. ${ }^{9-12}$ Cambria and colleagues reported that only 18 of 325 patients with spontaneous aortic dissections had concurrent degenerative aneurysmal disease. ${ }^{9}$ Of these 18 patients, 5 patients had history of a previously repaired AAA before suffering an acute aortic dissection. Only five patients suffered acute aortic dissections separate from a known infrarenal AAA. A recent study by Lee et al. has better defined the natural history of patients presenting with concurrent thoracic aortic dissections in the setting of AAAs. ${ }^{13}$ In this series, 12 patients initially presented with an acute aortic dissection and a concurrent AAA. This may pose a clinically challenging dilemma since these patients may exhibit abdominal pain due to their acute dissection, not their AAA. In contrast, impending AAA rupture may be difficult to discern from an acute aortic dissection. Anand et al. suggested that even in the setting of an acute aortic dissection, these aneurysms may be repaired safely with 
Table VI. In-hospital complications (preoperative and postoperative)

\begin{tabular}{lllr}
\hline Complication & Group I $[n(\%)]$ & Group II $[n(\%)]$ & $p$ \\
\hline New neurologic deficit & $2(4.7)$ & $200(23.2)$ & 0.004 \\
CVA/coma/spinal cord ischemia & $3(7)$ & $146(17.9)$ & 0.07 \\
Myocardial ischemia & $3(7.1)$ & $79(9)$ & $>0.99$ \\
Myocardial infarction & $1(2.4)$ & $41(4.7)$ & 0.72 \\
Mesenteric ischemia/infarction & $4(9.8)$ & $48(5.5)$ & 0.28 \\
Acute renal failure & $9(21.4)$ & $160(18.1)$ & 0.59 \\
Extension of dissection & $2(4.9)$ & $23(10.2)$ & 0.42 \\
Hypotension & $7(16.7)$ & $115(13.1)$ & 0.15 \\
Cardiac tamponade & $1(2.4)$ & $92(10.6)$ & 0.04 \\
Limb ischemia & $0(0)$ & & 0.03 \\
\hline
\end{tabular}

CVA, cerebrovascular accident.

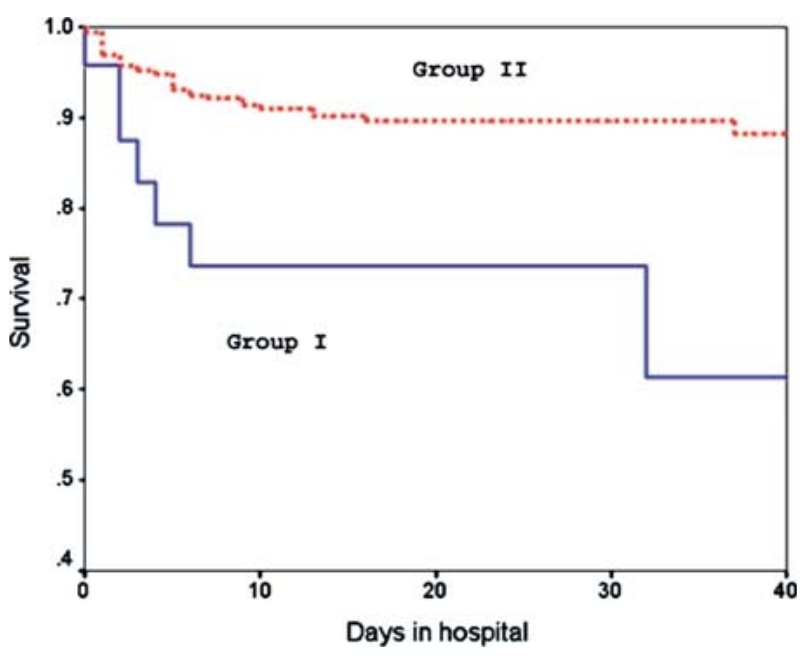

Fig. 1. Kaplan-Meier survival curve for patients presenting primarily with (Group I) or without (Group II) abdominal pain in the setting of acute type B thoracic aortic dissections. Log-rank test, $p=0.003$.

excellent long-term survival. ${ }^{14}$ In the present study, only two patients presented with a known aortic aneurysm, which suggests that this is likely not the cause of the excessive mortality seen in group I.

Another possible vascular etiology for abdominal pain in the setting of an acute aortic dissection is malperfusion of the visceral branches originating off of the abdominal aorta. ${ }^{15-17}$ While not reaching statistical significance in this study, this mechanism may be clinically important as nearly $10 \%$ of patients in group I developed mesenteric ischemia or infarction and it may have been undiagnosed in others. This suggests that perhaps some subclinical malperfusion syndrome may be occurring. The anatomy and radiologic diagnosis of visceral vessel compromise has been well described. Williams et al., using aortography, intravascular ultrasound, and mannometry, described two types of visceral artery luminal compromise in patients with acute aortic dissections. ${ }^{15}$ A static dissection flap occurs when the aortic dissection intersects the vessel origin and narrows its lumen. In contrast, a dynamic dissection seems to spare the vessel origin, but the flap compresses the true lumen at or above the vessel origin, thus functionally occluding the origin. A recent study documented a significant increase in mortality in patients with mesenteric ischemia in patients with acute type B aortic dissections. ${ }^{18}$ When malperfusion of the visceral vessels was specifically identified, $15.8 \%$ of all deaths in that large series had mesenteric ischemia. After adjusting for age and gender, branch vessel involvement was found to be an independent risk factor for death (odds ratio, 2.9; $p=0.02$ ).

Limitations of the present study include the relative small number of patients in the test group $(N=46)$ compared to the control group. Therefore, a type II statistical error is possible. While standard definitions were used, details surrounding diagnostic and therapeutic management of these patients were limited by the study's retrospective nature and the observation that each center managed patients individually and not by protocol. For example, no standardization occurred in the treatment of patients with type B dissections who required aortic or branch fenestration ${ }^{19}$ or primary thoracic aortic stent-grafting ${ }^{20,21}$ for mesenteric or renal ischemia. In addition, and importantly, ascertainment of patients to be included in group I with primary abdominal pain was by definition quite subjective.

Despite these limitations, the present study confirms the deadly nature of acute thoracic aortic dissections presenting in an atypical fashion, namely with primary abdominal pain. This study further underscores the importance of maintaining a high index of suspicion for an acute aortic dis- 


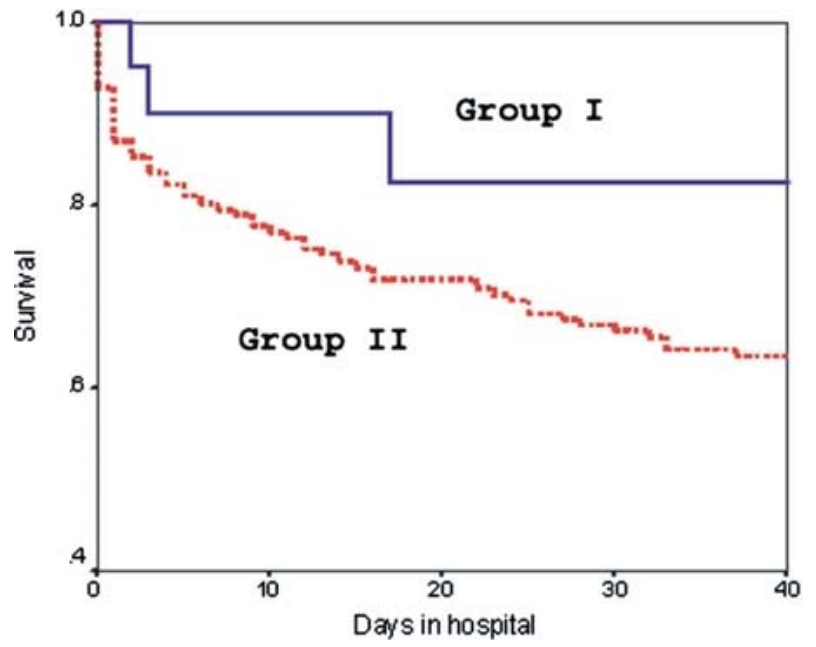

Fig. 2. Kaplan-Meier survival curve for patients presenting primarily with (Group I) or without (Group II) abdominal pain in the setting of acute type A thoracic aortic dissections. Log-rank test, $p=0.08$.

section in patients presenting with abdominal pain, as there are few other physical signs and symptoms that aid in the diagnosis of this lethal disease.

We thank Jeanna $V$. Cooper, MS, Jianming Fang, MD, and Dean E. Smith, PhD, University of Michigan Ann Arbor, MI, for data management and biostatistical support. The International Registry of Acute Aortic Dissection (IRAD) is supported by grants from the University of Michigan Health System and the Varbedian Fund for Aortic Research.

APPENDIX I. The International Registry of Acute Aortic Dissection (IRAD) Investigators

Co-Principal Investigators: Kim A. Eagle, MD, University of Michigan, Ann Arbor, Michigan USA; Eric M. Isselbacher, MD, Massachusetts General Hospital, Boston, Massachusetts, USA; Christoph A. Nienaber, MD, University of Rostock, Rostock, Germany.

Co-Investigators: Eduardo Bossone, MD, National Research Council, Lecce, Italy; Arturo Evangelista, MD, Hospital General Universitari Vall d'Hebron, Barcelona, Spain; Rosell Fattori, MD, University Hospital S. Orsola, Bologna, Italy; Dan Gilon, MD, Hadassah University Hospital, Jerusalem, Israel; Stuart Hutchison, MD, St. Michael's Hospital, Toronto, Ontario, Canada; James L. Januzzi, MD,
Massachusetts General Hospital, Boston, Massachusetts, USA; Alfredo Llovet, MD, Hospital Universitario " 12 de Octubre", Madrid, Spain; Rajendra H. Mehta, MD, MS, University of Michigan, Ann Arbor, Michigan, USA; Truls Myrmel, MD, Tromsø University Hospital, Tromsø, Norway; Patrick O'Gara, MD, and Joshua Beckman, MD, Brigham and Women's Hospital, Boston, Massachusetts, USA; Jae K. Oh, MD, Mayo Clinic, Rochester, Minnesota, USA; Linda A. Pape, MD, University of Massachusetts Hospital, Worcester, Massachusetts, USA; Udo Sechtem, MD, Robert-Bosch Krankenhaus, Stuttgart, Germany, Toru Suzuki, MD, University of Tokyo, Tokyo, Japan; Santi Trimarchi, MD, Istituto Policlinico San Donato, San Donato, Italy.

\section{REFERENCES}

1. National Hospital Discharge Survey Data. [database online @ http://www.cdc.gov/nchs/data/series/sr_13/sr13_153.pdf ]. Hyattsville, MD: National Center for Health Statistics; 2002. Updated November, 2002.

2. Klompas M. Does this patient have an acute thoracic aortic dissection? JAMA 2002;17:2262-2272.

3. Hagan PG, Nienaber CA, Isselbacher EM, et al. The International Registry of Acute Aortic Dissection (IRAD): new insights into an old disease. JAMA 2000;283:897-903.

4. Meszaros I, Morocz J, Szlavi J, et al. Epidemiology and clinicopathology of aortic dissection. Chest 2000;117:12711278.

5. Sullivan PR, Wolfson AB, Leckey RD, et al. Diagnosis of acute thoracic aortic dissection in the emergency department. Am J Emerg Med 2000;18:46-50.

6. Viljanen T. Diagnostic difficulties in aortic dissection: retrospective study of 89 surgically treated patients. Ann Chir Gynaecol 1986;75:328-332.

7. Eagle KA, Quertermous T, Kritzer GA, et al. Spectrum of conditions initially suggesting acute aortic dissection but with negative aortograms. Am J Cardiol 1986;57:322-326.

8. Ismil ML, Hussien M. Unusual presentation of dissecting aortic aneurysm. Int J Clin Pract 1999;53:389-390.

9. Cambria RP, Brewster DC, Moncure AC, et al. Spontaneous aortic dissection in the presence of coexistent or previously repaired atherosclerotic aortic aneurysm. Ann Surg 1988; 208:619-625.

10. Roberts CS, Roberts WC. Combined thoracic aortic dissection and abdominal aortic fusiform aneursym. Ann Thorac Surg 2001;52:537-540.

11. Gugulakis AG, Matsagas MI, Vasdekis SN, et al. Rupture of an abdominal aortic aneurysm following acute descending thoracic aortic dissection, case report. J Cardiovasc Surg 1998;39:583-585.

12. Jacobs DL, Freischlag JA, Seabrook GR, et al. Acute aortic dissection into a preexisting abdominal aortic aneurysm. Ann Vasc Surg 1984;8:491-495.

13. Lee JJ, Dimick JB, Williams DM, et al. Existence of abdominal aortic aneurysms in patients with thoracic aortic dissections. J Vasc Surg 2003;38:671-675.

14. Anand RJ, Williams DM, Proctor MC, et al. Operative mortality for elective abdominal aortic aneurysm repair is not increased by the presence of a previous or concurrent 
thoracic or thoracoabdominal aortic dissection. J Vasc Surg 2002;36:690-695.

15. Williams DM, Lee DY, Hamilton BH, et al. The dissected aorta: part III. Anatomy and radiologic diagnosis of branchvessel compromise. Radiology 1997;203:37-44.

16. Vedantham S, Picus D, Sanchez LA, et al. Percutaneous management of ischemic complications in patients with type-B aortic dissection. J Vasc Interv Radiol 2003;14:18193.

17. Harms J, Hess U, Cavallaro A, et al. The abdominal aortic fenestration procedure in acute thoraco-abdominal aortic dissection with aortic branch artery ischemia. J Cardiovasc Surg 1998;39:273-280.
18. Suzuki $\mathrm{T}$, Mehta $\mathrm{RH}$, Ince $\mathrm{H}$, et al. Clinical profiles and outcomes of acute type B aortic dissection in the current era: lessons from the International Registry of Aortic Dissection (IRAD). Circulation 2003;108 (Suppl II):II-312-II-317.

19. Slonim SM, Nyman UR, Semba CP, et al. True lumen obliteration in complicated aortic dissection: endovascular treatment. Radiology 1996;201:161-166.

20. Dake MD, Kato N, Mitchell RS, et al. Endovascular stentgraft placement of acute aortic dissection. N Engl J Med 1999;340:1546-1552.

21. Nienaber CA, Fattori R, Lund G, et al. Nonsurgical reconstruction of thoracic aortic dissection by stent-graft placement. N Engl J Med 1999;340:1539-1545. 